\title{
Knockdown of IncRNA-UCA1 inhibits the proliferation and migration of melanoma cells through modulating the miR-28-5p/HOXB3 axis
}

\author{
CHAOFEI HAN, FENGJIE TANG, JIA CHEN, DAN XU, XIONG LI, \\ YANGCHENG XU, SHAOHUA WANG and JIANDA ZHOU \\ Department of Burn and Plastic Surgery, Third Xiangya Hospital of Central South University, \\ Changsha, Hunan 410013, P.R. China
}

Received March 29, 2018; Accepted December 28, 2019

DOI: $10.3892 /$ etm.2019.7421

\begin{abstract}
Long non-coding RNA urothelial carcinoma-associated 1 (UCA1) functions as an oncogene in different human cancers, including melanoma. However, the molecular mechanism of UCA1 underlying melanoma progression still remains largely unknown. In the present study, reverse transcription quantitative polymerase chain reaction and western blot analyses were used to examine the mRNA and protein expression levels, respectively. Cell Counting Kit-8 and wound healing assays were conducted to study cell proliferation and migration, respectively. A luciferase reporter assay was used to confirm the targeting relationship. It was demonstrated that UCA1 expression was increased in melanoma tissues and cell lines. In addition, UCA1 expression was higher in melanoma tissues at stage III-IV than in tissues at stage I-II. Inhibition of UCA1 expression markedly reduced melanoma cell proliferation and migration. Further investigation revealed that UCA1 functioned in melanoma cells through directly binding with microRNA (miR)-28-5p. The expression of miR-28-5p was significantly reduced in melanoma tissues and had an inverse correlation with UCA1 expression. In addition, miR-28-5p expression was higher in melanoma tissues at advanced stages than in stage I-II tissues. Furthermore, homeobox (HOX)B3 was identified as a target gene of miR-28-5p in melanoma cells, and HOXB3 overexpression reversed the suppressive effects of UCA1 downregulation on melanoma cell proliferation and migration. Finally, HOXB3 was upregulated in melanoma tissues compared with its expression in adjacent tissues, and HOXB3 expression was increased in melanoma tissues at advanced stages. Taken together, the regulatory network of
\end{abstract}

Correspondence to: Professor Jianda Zhou, Department of Burn and Plastic Surgery, Third Xiangya Hospital of Central South University, 138 Tongzipo Road, Changsha, Hunan 410013, P.R. China E-mail: zhoujianda@csu.edu.cn

Key words: melanoma, microRNAs, long non-coding RNAs, homeobox B3 the UCA1/miR-28-5p/HOXB3 axis in melanoma was demonstrated for the first time in the present study, expanding the understanding of the molecular mechanism underlying melanoma progression. Future studies may further confirm the function of this signaling pathway in vivo.

\section{Introduction}

Melanoma is the most prevalent malignant skin cancer. Melanoma ranks as the fifth most prevalent cancer in males and the seventh most prevalent cancer in females, causing $\sim 80 \%$ skin cancer-associated mortalities worldwide $(1,2)$. Incidence rates of malignant melanoma have increased significantly since 1992 with an overall $45 \%$ increase and estimated $3.1 \%$ annual percent change $(1,2)$. Therefore, exploring the molecular mechanism during melanoma progression is urgently required as it may benefit the development of effective strategies for melanoma treatment.

Long non-coding RNAs (lncRNAs) are a newly discovered group of small non-coding RNAs $>200$ nucleotides in length, comprising $80 \%$ of non-coding RNAs (3-5). In recent years, a large number of lncRNAs have been demonstrated to participate in various cellular biological processes, such as cell proliferation, differentiation, apoptosis, cell cycle, motility, and tumourigenesis (6,7). A number of lncRNAs have been identified as oncogenes or tumor suppressors in human cancers, including melanoma $(8,9)$. For instance, the lncRNA growth arrest-specific 5 serves an inhibitory role in melanoma via regulating gelatinase $\mathrm{A}$ and $\mathrm{B}$ both in vitro and in vivo (10). The IncRNA BRAF-activated non protein-coding RNA promotes melanoma cell proliferation by regulating mitogen-activated protein kinase pathway activity (11).

Among cancer-related lncRNAs, urothelial cancer-associated 1 (UCA1) generally serves a tumor-promoting role $(12,13)$. Tian et al (14) previously reported that UCA1 was significantly upregulated in melanoma tissues compared with its expression in paired adjacent non-tumor tissues, and melanomas at advanced stages exhibited higher UCA1 expression than tumors at early stages. Furthermore, previous studies have demonstrated that UCA1 functions as an oncogene in certain common human cancers through directly interacting with its 
target microRNAs (miRNAs or miRs) and further affecting the protein expression of the downstream target genes $(15,16)$. For instance, UCA1 promotes the proliferation and migration of pancreatic cancer cells through regulating the miR-96/forkhead box protein (FOX)O3 axis (17). In addition, UCA1 promotes the migration and epithelial-mesenchymal transition of bladder cancer cells by regulating the miR-143/high mobility group box 1 pathway (12). In melanoma, UCA1 promotes cancer cell proliferation, cell cycle progression and migration via modulation of the miR-507-FOXM1 axis (18). However, whether other miRNAs and downstream proteins are also associated with UCA1-mediated melanoma cells remains unclear.

miR-28-5p has been demonstrated to serve different roles in different cancer types $(19,20)$. For instance, miR-28-5p promotes ovarian cancer progression through inhibition of NEDD4 binding protein 1 (20). In contrast, miR-28-5p is downregulated in colorectal cancer, and overexpression of miR-28-5p exhibits suppressive effects on colorectal cancer cell proliferation, migration and invasion in vitro, as well as tumor growth in vivo (19). furthermore, homeobox (HOX)B3is hypothesized to be a direct target gene of miR-28-5p, and the expression of HOXB3 is regulated by miR-28-5p in colorectal cancer cells (19). However, the detailed role of miR-28-5p and HOXB3 in melanoma remains unclear. Therefore, the present study aimed to explore the molecular mechanism of UCA1 underlying melanoma cell proliferation and migration.

\section{Materials and methods}

Tissue samples. The present study was approved by the Research Ethics Committee of Third Xiangya Hospital (Changsha, China). A total of 22 melanoma tumors and matched adjacent non-tumor tissues were collected from primary melanoma patients at the Department of Burn and Plastic Surgery, Third Xiangya Hospital of Central South University (Changsha, China) between April 2014 and May 2017. These patients included 10 males and 12 females from 34-60 years old with a mean age of 48.3 years. In total, 12 I-II stage cases and 10 III-IV stage cases were included. No patient recruited for the present study had received adjuvant treatment prior to surgical resection. Written informed consent was obtained from all patients.

Cell culture. Normal human epidermal melanocyte HEMa-LP cells and human melanoma cell lines, including A375, SK-MEL-2 and SK-MEL-28, were obtained from the Cell Bank of the Chinese Academy of Science (Shanghai, China). Cells were cultured in Dulbecco's modified Eagle's medium (Thermo Fisher Scientific, Inc., Waltham, MA, USA) with $10 \%$ fetal bovine serum (Thermo Fisher Scientific, Inc.) and incubated at $37^{\circ} \mathrm{C}$ in a humidified atmosphere with $5 \%$ $\mathrm{CO}_{2}$.

Cell transfection. SK-MEL-28 cells were seeded $\left(1 \times 10^{5}\right.$ cells per well) into a 6-well plate and were transiently transfected with 50 nM UCA1 small interfering (si)RNA (siUCA1; cat. no. NR_015379), negative control siRNA (siNC; cat. no. SIC001), miR-28-5p mimic (cat. no. HMI0425), NC miR mimic (miR-NC; cat. no. HMC0002; all Sigma-Aldrich; Merck KGaA, Darmstradt, Germany), or co-transfected with
UCA1 siRNA and the HOBX3 expression plasmid or the blank pcDNA3.1 vector (Yearthbio Technology, Changsha, China) using Lipofectamine 2000 (Thermo Fisher Scientific, Inc.) according to the manufacturer's instruction. At $48 \mathrm{~h}$ following transfection, the cells were harvested, and the following assays were conducted.

Luciferase reporter gene assay. miRanda software version 1.0 (http://www.micro-RNA.org/) was used to predict potential UCA1-miR interactions. TargetScan software 7.2 (www. targetscan.org/) was used to predict the potential target genes of miR-28-5p. UCA1 sequences containing the wild-type (WT) or mutated (MT) miR-28-5p binding sites and HOXB3 3'UTR sequences containing the WT or MT miR-28-5p binding sites were subcloned into the pmiR-GLo luciferase reporter vector (Promega Corporation, Madison, WI, USA). SK-MEL-28 cells were co-transfected with WT or MT UCA1 reporter plasmids together with miR-28-5p mimic or miR-NC, or co-transfected with the WT or MT HOXB3 reporter plasmids together with miR-28-5p mimic or miR-NC using Lipofectamine 2000. At $48 \mathrm{~h}$ following transfection, luciferase activity was examined using the Dual Luciferase Reporter Assay system (Promega Corporation). The activity of firefly luciferase was normalized to the activity of Renilla luciferase.

Reverse transcription quantitative polymerase chain reaction $(R T-q P C R)$. Total RNA was extracted from tissues and, HEMa-LP, A375, SK-MEL-2 and SK-MEL-28 cells using TRIzol reagent (Thermo Fisher Scientific, Inc.,) and then reverse transcribed into cDNA using an OneStep RT-PCR kit (Qiagen, Inc., Valencia, CA, USA) according to the manufacturer's protocol. SYBR ${ }^{\circledR}$ Premix Ex Taq ${ }^{\mathrm{TM}}$ (Takara Biotechnology Co., Ltd., Dalian, China) was used to perform qPCR to determine UCA1, miR-28-5p and HOXB3 expression with an ABI 7300 Plus Fast Sequence Detection system (Applied Biosystems; Thermo Fisher Scientific, Inc.). The reaction was performed under the following conditions: $95^{\circ} \mathrm{C}$ for $3 \mathrm{~min}$ followed by 35 cycles at $95^{\circ} \mathrm{C}$ for $30 \mathrm{sec}$ and $60^{\circ} \mathrm{C}$ for $30 \mathrm{sec}$. The relative expression was calculated and normalized via the $2^{-\Delta \Delta \mathrm{Cq}}$ method (21). GAPDH and U6 were used as internal controls. The following primer sequences were utilized in these studies: miR-28-5p, 5'-AAGGAGCUCACA GUCUAUUGAG-3' and reverse 5'-CUCAAUAGACUGUGA GCUCCUU-3'; UCA1, forward 5'-CTCTCCATTGGGTTC ACCATTC-3' and reverse 5'-GCGGCAGGTCTTAAGAGA TGAG-3'; HOXB3, forward 5'-CCAGTGCCACTAGCAACA G-3' and reverse 5'-CGTTTGCCTCGACTCTTTCATC-3'. GAPDH, forward 5'-TGTTCGTCATGGGTGTGAAC-3' and reverse 5'-ATGGCATGGACTGTGGTCAT-3'; U6, forward 5'-CTCGCTTCGGCAGCACA-3' and reverse 5'-AACGCT TCACGAATTTGCGT-3'.

Cell proliferation analysis. Cell Counting Kit (CCK)-8 assays (Dojindo Molecular Technologies, Inc., Kumamoto, Japan) were conducted to study cell proliferation following the manufacturer's protocol. Following transfection for $48 \mathrm{~h}$, SK-MEL-28 cells (5,000 cells per well) were seeded onto 96-well plates, and cell proliferation was measured at 0,24 , 48 and $72 \mathrm{~h}$ by using the CCK-8. Absorbance was detected at optical density of $450 \mathrm{~nm}$ using a spectrophotometer. 
Cell migration analysis. Transfected SK-MEL-28 cells were seeded in 6-well plates and incubated at $37^{\circ} \mathrm{C}$ until $90 \%$ confluence. Following serum starvation at $37^{\circ} \mathrm{C}$ for $24 \mathrm{~h}$, wounds were created using a $100 \mu \mathrm{l}$ pipette tip. Wound healing was observed and photographed at 0 and $24 \mathrm{~h}$.

Western blotting. Total proteins were extracted from SK-MEL-28 cells using RIPA lysis buffer (Beyotime Institute of Biotechnology, Beijing, China). The protein concentration was measured using a bicinchoninic acid protein assay kit (Thermo Fisher Scientific, Inc.). Proteins (50 $\mu \mathrm{g}$ per lane) were separated by SDS-PAGE on $10 \%$ gels and transferred onto polyvinylidene difluoride membranes. Then membranes were incubated with the following antibodies: HOXB3 (1:500; cat. no. ab83404), MMP-9 (1:500; cat. no. ab73734) and GAPDH (1:500; cat. no. ab9485) for $3 \mathrm{~h}$ at room temperature, and horseradish peroxidase-conjugated secondary antibody (1:5,000; cat. no. ab6721; all Abcam, Cambridge, UK) for $1 \mathrm{~h}$ at room temperature. GAPDH was used as the internal control. The protein bands were detected using an Enhanced Chemiluminescence Western Blotting kit (Pierce; Thermo Fisher Scientific, Inc.) and quantified using Image Lab analysis software version 3.1 (Bio-Rad Laboratories, Inc., Hercules, CA, USA).

Statistical analysis. Data are presented as the mean \pm standard deviation. SPSS 19.0 software (IBM Corp., Armonk, NY, USA) was used to perform statistical analysis. Student's t-test and one-way analysis of variance with Tukey's post hoc test were used. Pearson correlation analysis was performed to examine the correlation between UCA1 and miR-28-5p expression in melanoma tissues. $\mathrm{P}<0.05$ was considered to indicate a statistically significant difference.

\section{Results}

UCA1 is upregulated in melanoma tissues and cell lines. In the present study, RT-qPCR was initially performed to examine UCA1 expression in a total of 22 melanoma issues and their matched adjacent non-tumor tissues. As indicated in Fig. 1A, UCA1 expression was significantly higher in melanoma tissues than in adjacent non-tumor tissues. Furthermore, UCA1 expression was significantly higher in melanoma tissues at stage III-IV than in tissues at stage I-II (Fig. 1B). Consistently, UCA1 was also significantly upregulated in human melanoma cell lines (A375, SK-MEL-2 and SK-MEL-28) compared with its expression in normal human epidermal melanocyte HEMa-LP cells (Fig. 1C). These findings suggest that UCA1 is upregulated in melanoma.

Knockdown of UCA1 expression suppresses SK-MEL-28 cell proliferation and migration. The role of UCA1 in the regulation of melanoma cell proliferation and migration was then investigated. Given that SK-MEL-28 cells exhibited the highest expression of UCA1, these cells were transfected with UCA1 siRNA to knockdown its expression. Following transfection, UCA1 expression levels were significantly reduced in the siUCA1 group compared with those in the siNC group (Fig. 2A). CCK-8 and wound healing assays were conducted to evaluate the effects of UCA1 downregulation on SK-MEL-28 cell proliferation and migration, respectively. CCK-8 assay data revealed that inhibition of UCA1 expression caused a significant reduction in SK-MEL-28 cell proliferation (Fig. 2B). Similarly, knockdown of UCA1 expression significantly inhibited SK-MEL-28 cell migration (Fig. 2C). Thus, downregulation of UCA1 effectively inhibited SK-MEL-28 cell proliferation and migration.

miR-28-5p is a target of UCA1 in melanoma cells. LncRNAs act as sinks for pools of miRNAs to regulate the downstream target genes $(15,16)$. Therefore, miRanda software was used to predict potential UCA1-miR interactions. The data demonstrated that miR-28-5p harbors a potential binding site for UCA1 (Fig. 3A). It was then demonstrated that UCA1 knockdown led to significant upregulation of miR-28-5p expression in SK-MEL-28 cells (Fig. 3B). To further confirm their targeting relationship, luciferase reporter gene plasmids were constructed containing UCA1-WT and UCA1-MT binding sites with miR-28-5p (Fig. 3A). SK-MEL-28 cells were then co-transfected with miR-NC or miR-28-5p and UCA1-WT or UCA1-MT luciferase reporter gene plasmids. Dual luciferase assay results revealed that miR-28-5p significantly reduced the luciferase activity of the UCA1-WT luciferase reporter gene plasmid but did not affect the luciferase activity of the UCA1-MT luciferase reporter gene plasmid (Fig. 3C). Furthermore, miR-28-5p was significantly downregulated in melanoma tissues compared with that in adjacent non-tumor tissues (Fig. 3D), and high expression of UCA1 exhibited a negative correlation with low miR-28-5p expression in melanoma tissues (Fig. 3E). In addition, miR-28-5p expression was significantly lower in melanoma stage III-IV tissues than in stage I-II tissues (Fig. 3F). Finally, miR-28-5p was also significantly downregulated in melanoma cell lines compared with the expression in normal human epidermal melanocyte HEMa-LP cells (Fig. 3G).

miR-28-5p is associated with UCA1-mediated melanoma cell proliferation and migration. It was investigated whether miR-28-5p is associated with the UCA1-mediated proliferation and migration of melanoma cells. As it was demonstrated that miR-28-5p was upregulated following knockdown of UCA1 expression, siUCA1-transfected SK-MEL-28 cells were then transfected with miR-28-5p inhibitor to reduce its expression. Following transfection, miR-28-5p expression levels were significantly decreased in the siUCA1+miR-28-5p inhibitor group compared with those in the siUCA1+NC inhibitor group (Fig. 4A). Furthermore, CCK-8 assay and wound healing assay data demonstrated that the proliferation and migration capacities of cells were significantly increased in the siUCA1+miR-28-5p inhibitor group compared with those in the siUCA1+NC inhibitor group (Fig. 4B and C). These results suggest that knockdown of UCA1 expression inhibits SK-MEL-28 cell proliferation and migration via upregulation of miR-28-5p.

HOXB3 is a target gene of miR-28-5p in SK-MEL-28 cells. The downstream effector of miR-28-5p in SK-MEL-28 cells was then investigated, and Targetscan software predicted that HOXB3 was a target gene of miR-28-5p 

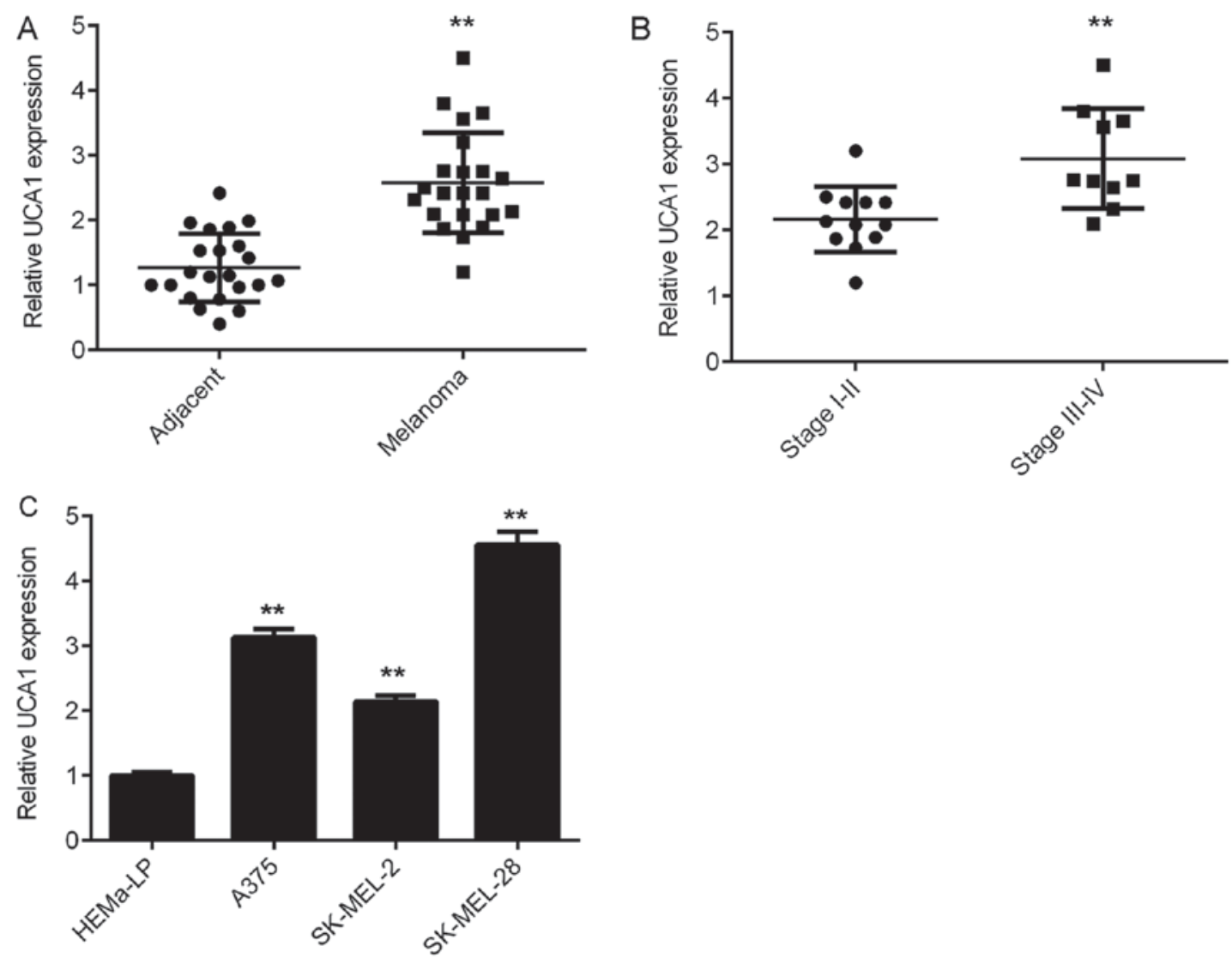

Figure 1. UCA1 is upregulated in melanoma tissues and cell lines. (A) RT-qPCR was used to examine the expression of UCA1 in 21 melanoma issues and their matched adjacent non-tumor tissues. ${ }^{* *} \mathrm{P}<0.01$ vs. adjacent. (B) RT-qPCR data demonstrated that the expression of UCA1 was higher in melanoma tissues at stage III-IV when compared with that in tissues at stage I-II. ${ }^{* *} \mathrm{P}<0.01$ vs. stage I-II. (C) RT-qPCR was used to examine the expression of UCA1 in human melanoma cell lines (A375, SK-MEL-2 and SK-MEL-28) and in normal human epidermal melanocyte HEMa-LP cells. "P<0.01 vs. HEMa-LP. UCA1, urothelial carcinoma-associated 1; RT-qPCR, reverse transcription-quantitative polymerase chain reaction.
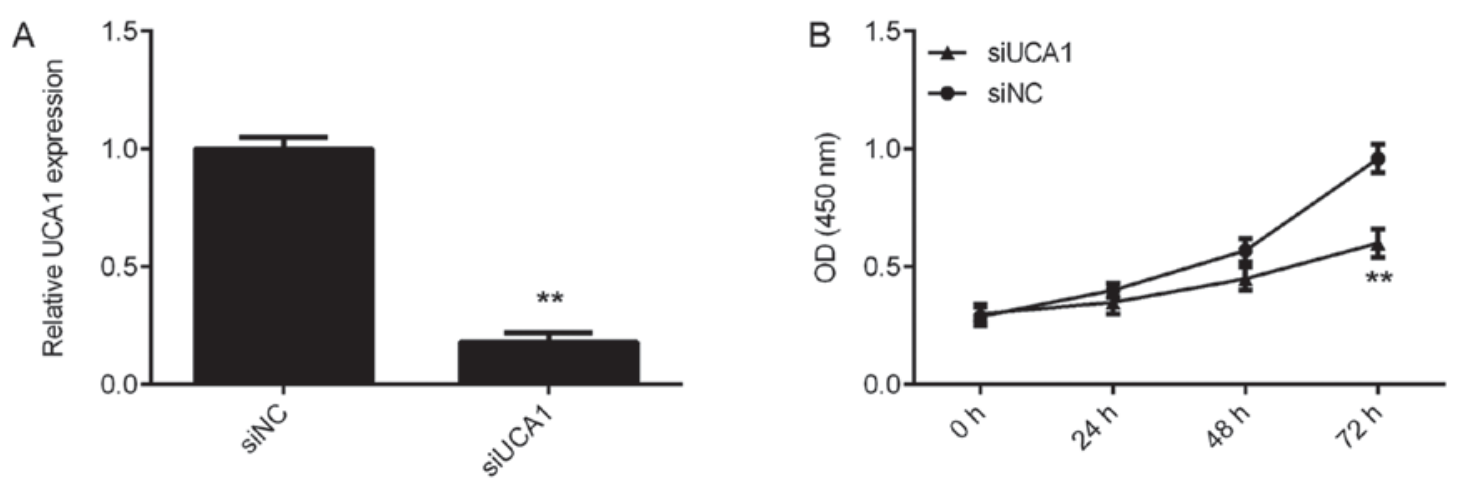

C
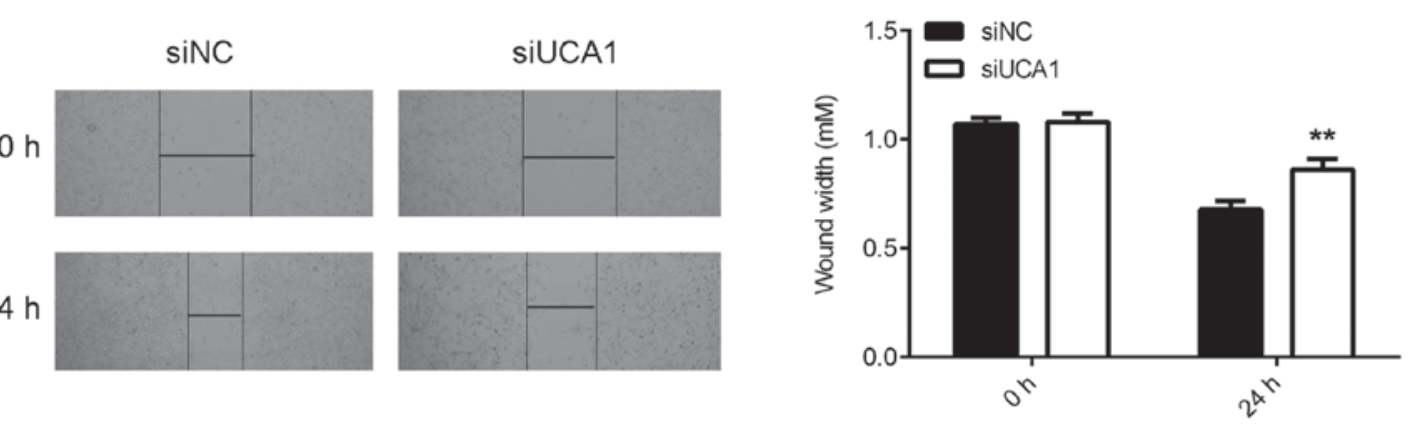

Figure 2. Knockdown of UCA1 expression suppressed the proliferation and migration of SK-MEL-28 cells. SK-MEL-28 cells were transfected with siUCA1 and siNC, respectively. Following transfection, (A) reverse transcription-quantitative polymerase chain reaction was used to examine the expression of UCA1. (B) Cell counting kit-8 analysis and (C) wound healing assay were used to evaluate the cell proliferation and migration, respectively. ${ }^{* *} \mathrm{P}<0.01 \mathrm{vs}$. siNC. UCA1, urothelial carcinoma-associated 1; si, small interfering RNA; NC, negative control; OD, optical density. 
A

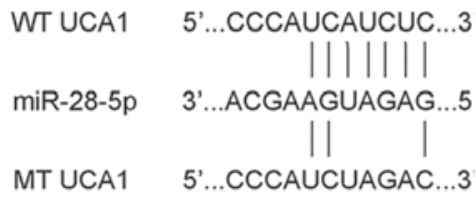

$\mathrm{D}$

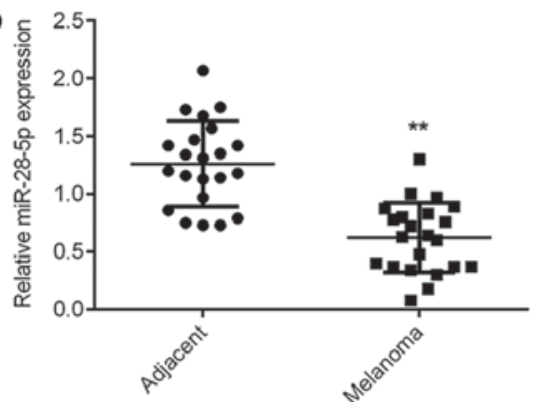

G

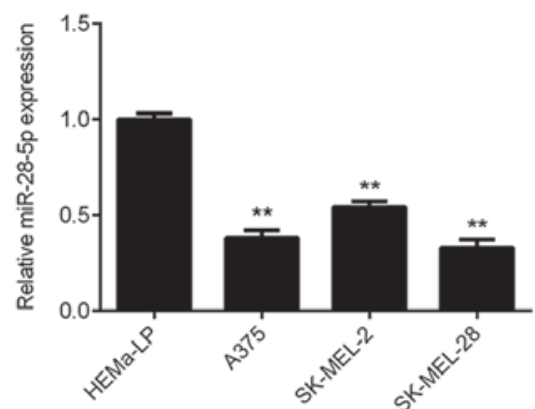

B

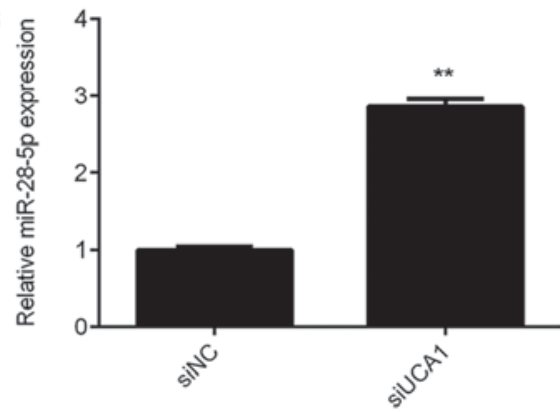

$E$

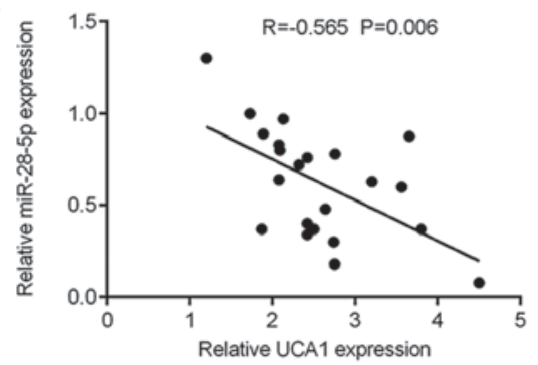

$c$

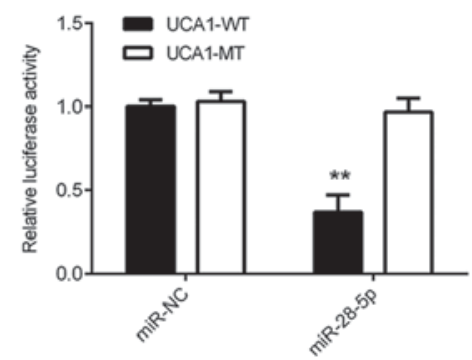

$\mathrm{F}$

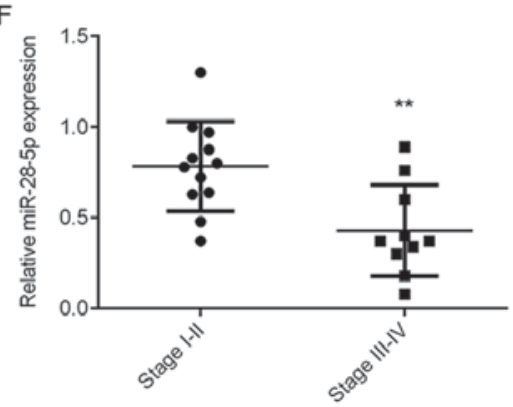

Figure 3. miR-28-5p is a target of UCA1 in melanoma cells. (A) WT and MT UCA1 binding sites with miR-28-5p are presented. (B) RT-qPCR data indicated that knockdown of UCA1 led to an upregulation of miR-28-5p expression in SK-MEL-28 cells. ${ }^{* *} \mathrm{P}<0.01$ vs. siNC. (C) miR-28-5p significantly reduced the luciferase activity of the UCA1-WT luciferase reporter gene plasmid, but did not affect the luciferase activity of the UCA1-MT luciferase reporter gene plasmid. ${ }^{* *} \mathrm{P}<0.01$ vs. miR-NC. (D) RT-qPCR was used to examine the expression of miR-28-5p in 22 melanoma issues and their matched adjacent non-tumor tissues. ${ }^{* *} \mathrm{P}<0.01$ vs. adjacent. (E) The expression of UCA1 exhibited a negative association with the expression of miR-28-5p in melanoma tissues. (F) The expression of miR-28-5p was lower in melanoma tissues at stage III-IV when compared with that in tissues at stage I-II. ${ }^{* *} \mathrm{P}<0.01$ vs. stage I-II. (G) RT-qPCR was used to examine the miR-28-5p expression in human melanoma cell lines (A375, SK-MEL-2 and SK-MEL-28) and in normal human epidermal melanocyte HEMa-LP cells. ${ }^{* *} \mathrm{P}<0.01$ vs. HEMa-LP. miR, microRNA; UCA1, urothelial carcinoma-associated 1; WT, wild type; MT, mutant type; RT-qPCR, reverse transcription-quantitative polymerase chain reaction; si, small interfering RNA; NC, negative control.

(Fig. 5A). To confirm the prediction, WT-HOXB3-3'UTR and MT-HOXB3-3'UTR luciferase reporter plasmids were generated (Fig. 5A). Luciferase reporter gene assay data indicated that miR-28-5p significantly reduced the luciferase activity of the HOXB3-WT luciferase reporter gene plasmid but did not affect the luciferase activity of the HOXB3-MT luciferase reporter gene plasmid (Fig. 5B), indicating that miR-28-5p directly binds to the 3'UTR of HOXB3 mRNA in SK-MEL-28 cells. Furthermore, it was demonstrated that UCA1 knockdown significantly reduced HOXB3 expression, which was abolished by inhibition of miR-28-5p expression in SK-MEL-28 cells (Fig. 5C and D). These above findings suggest that UCA1 affects HOXB3 expression via miR-28-5p. In addition, it was demonstrated that HOXB3 was upregulated in melanoma tissues compared with its expression in adjacent normal tissues, and HOXB3 protein expression was high in tumor tissues at advanced stages compared with that in tissues at earlier stages (Fig. 5E and F).
HOXB3 acts as a downstream effector of UCA1 in SK-MEL-28 cells. It was then investigated whether HOXB3 acts as a downstream effector of UCA1 in SK-MEL-28 cells. As HOXB3 was downregulated following knockdown of UCA1 expression, siUCA1-transfected SK-MEL-28 cells were then transfected with the HOXB3 expression plasmid to upregulate its expression. Following transfection, HOXB3 mRNA and protein expression levels were significantly increased in the siUCA1+HOXB3 group compared with those in the siUCA1+blank group (Fig. 6A and B). Furthermore, CCK-8 assay and wound healing assay data revealed that the proliferation and migration capacities of cells were significantly increased in the siUCA1+HOXB3 group compared with those in the siUCA1+blank group (Fig. 6C and D). These findings indicate that HOXB3 overexpression significantly rescued the suppressive effects of UCA1 downregulation on the proliferation and migration of SK-MEL-28 cells. Taken together, these findings suggest that inhibition of UCA1 expression suppresses 

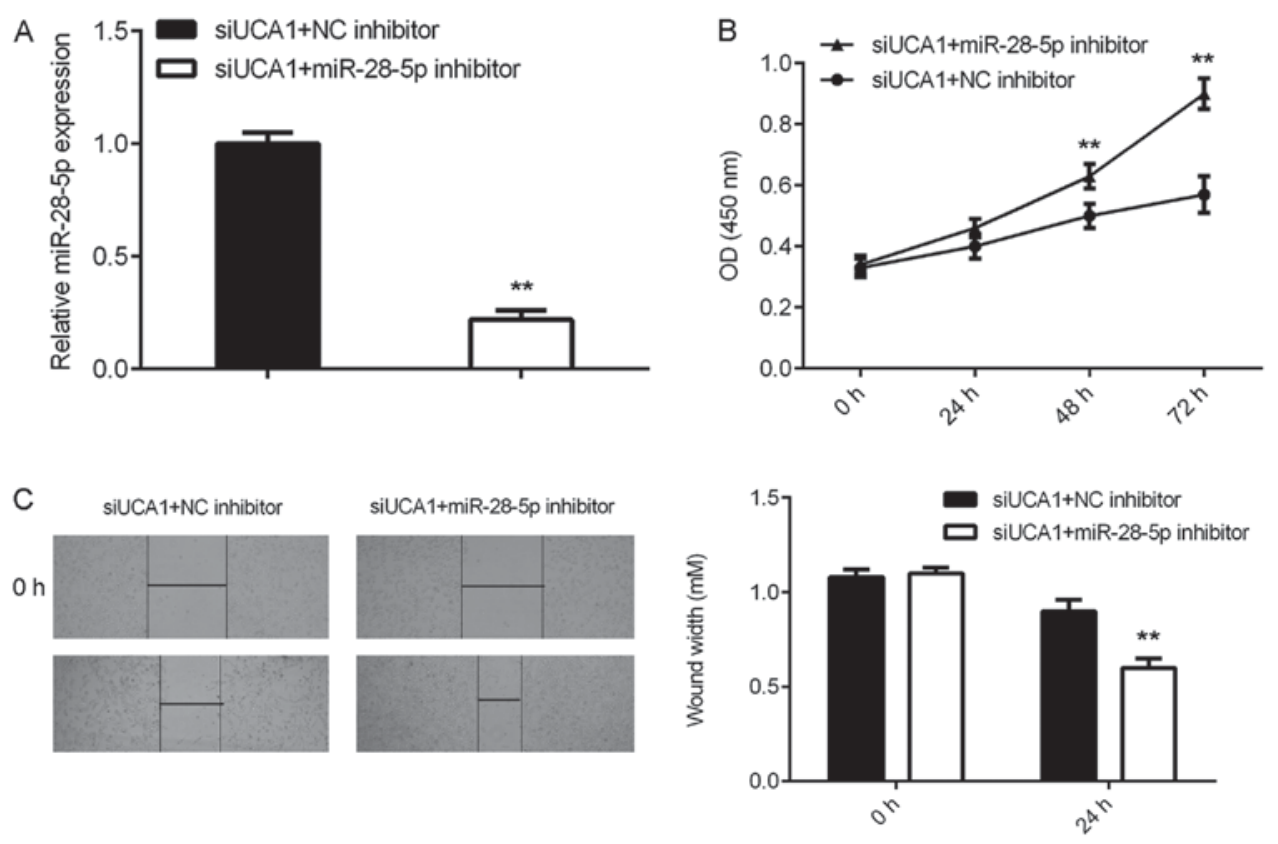

Figure 4. miR-28-5p is associated with UCA1-mediated proliferation and migration and melanoma cells. siUCA1 transfected SK-MEL-28 cells were transfected with miR-28-5p inhibitor or NC inhibitor. (A) Following transfection, reverse transcription-quantitative polymerase chain reaction was used to examine the expression of miR-28-5p. (B) Cell counting kit-8 analysis and (C) wound healing assay were used to evaluate the cell proliferation and migration. ${ }^{* *} \mathrm{P}<0.01$ vs. siUCA1+NC inhibitor. miR, microRNA; UCA1, urothelial carcinoma-associated 1; si, small interfering RNA; NC, negative control; OD, optical density.

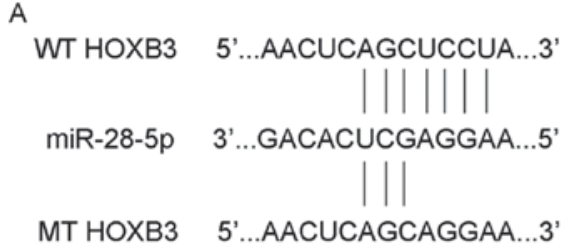

C
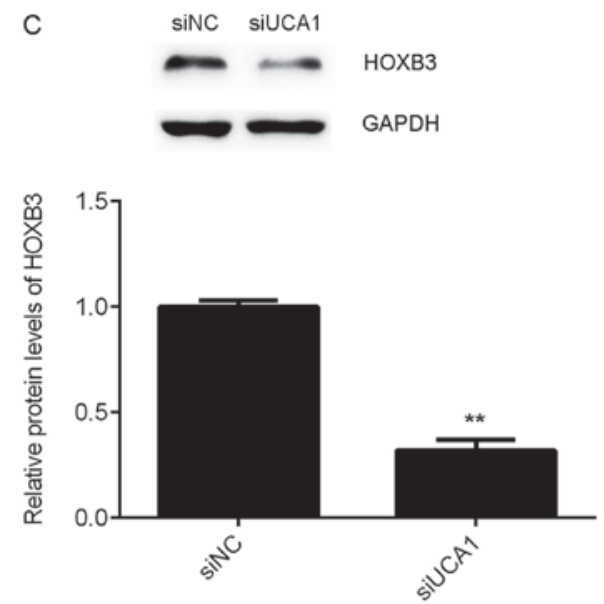

E

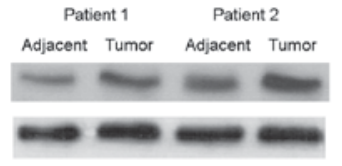

\section{HOXB3 \\ GAPDH}

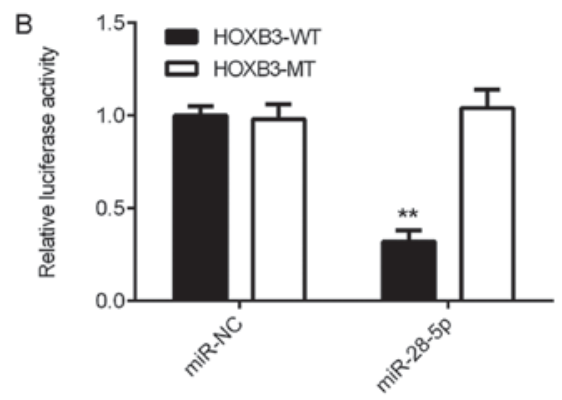

D
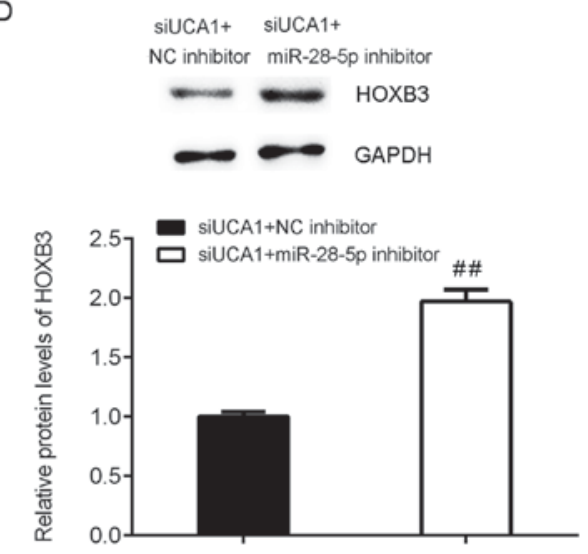

$\mathrm{F}$

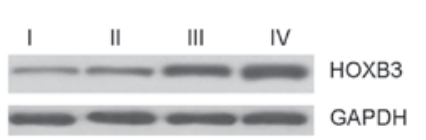

Figure 5. HOXB3 is a target gene of miR-28-5p in SK-MEL-28 cells. (A) WT and MT HOXB3 binding sites with miR-28-5p are presented. (B) miR-28-5p significantly reduced the luciferase activity of the HOXB3-WT luciferase reporter gene plasmid, but did not affect the luciferase activity of the HOXB3-MT luciferase reporter gene plasmid. ${ }^{* *} \mathrm{P}<0.01$ vs. miR-NC. (C and D) Knockdown of UCA1 decreased the mRNA and protein expression of HOXB3, which was abolished by inhibition of miR-28-5p expression in SK-MEL-28 cells. ${ }^{* *} \mathrm{P}<0.01$ vs. siUCA1. ${ }^{\# t} \mathrm{P}<0.01$ vs. siUCA1+NC inhibitor. (E) HOXB3 was upregulated in melanoma tissues compared with adjacent normal tissues. (F) The protein expression of HOXB3 was high in tumor tissues at advanced stages compared with that in tissues at stage I-II. HOX, homeobox; miR, microRNA; WT, wild type; MT, mutant type; NC, negative control; UCA1, urothelial carcinoma-associated 1; si, small interfering RNA. 
A

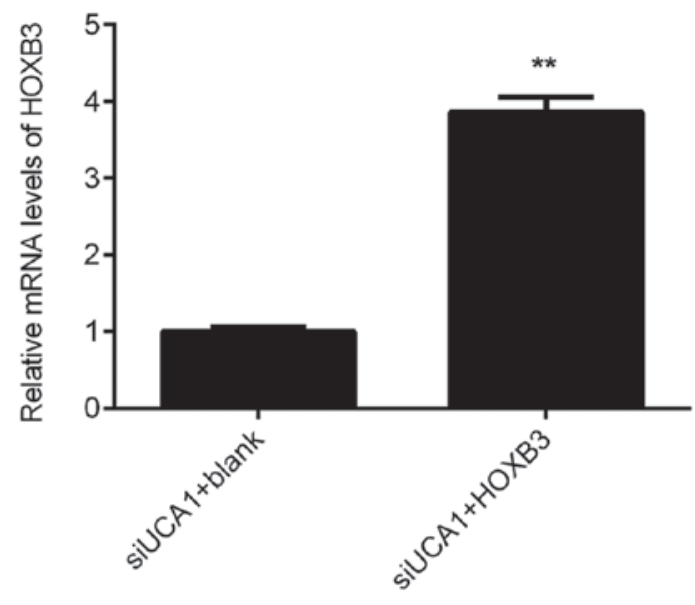

B

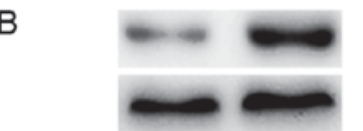

HOXB3

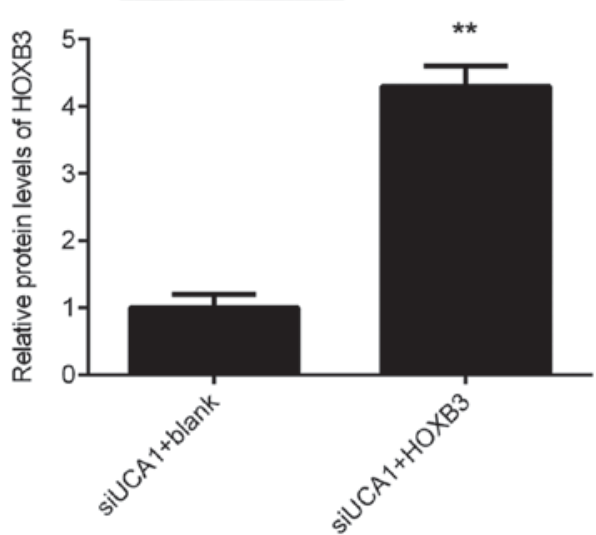

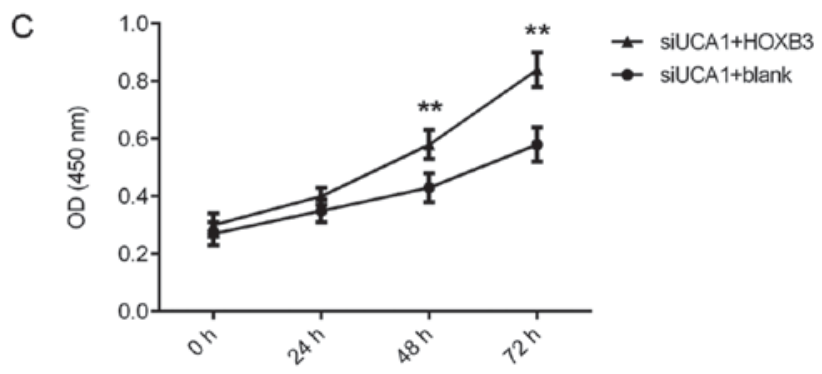

D
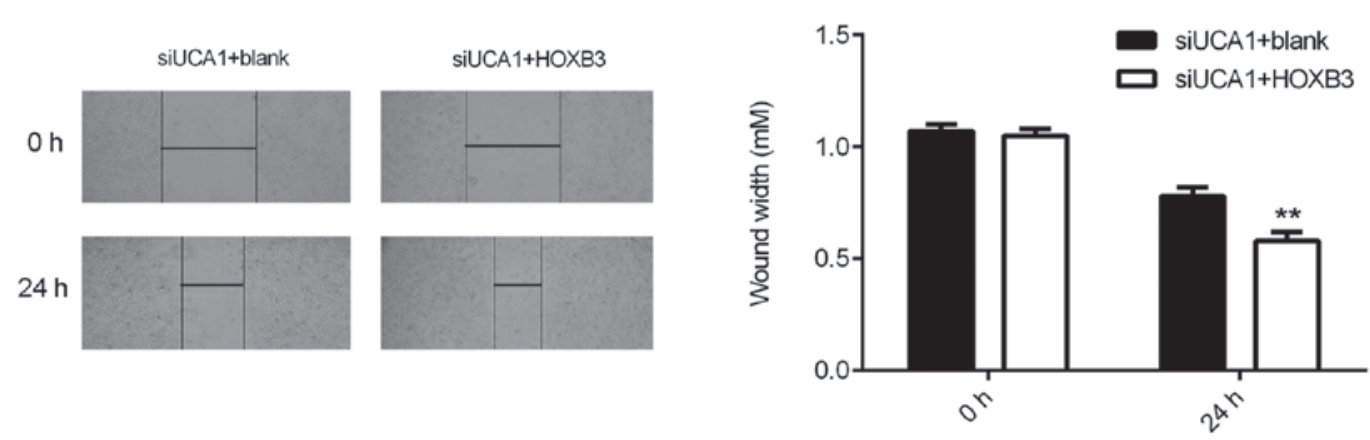

Figure 6. HOXB3 acts as a downstream effecter of UCA1 in SK-MEL-28 cells. The siUCA1 transfected SK-MEL-28 cells were then transfected with HOXB3 expression plasmid or blank vector. Following transfection, (A) reverse transcription-quantitative polymerase chain reaction and (B) western blotting were used to examine the mRNA and protein expression of HOXB3, respectively. (C) Cell counting kit-8 analysis and (D) wound-healing assays were used to evaluate the cell proliferation and migration. ${ }^{* *} \mathrm{P}<0.01$ vs. siUCA1+blank. HOX, homeobox; UCA1, urothelial carcinoma-associated 1 ; si, small interfering RNA; OD, optical density.

SK-MEL-28 cell proliferation and migration through modulating the miR-28-5p/HOXB3 axis.

\section{Discussion}

UCA1 serves a promoting role in different human cancers $(15,22)$, but the detailed regulatory mechanism of UCA1 underlying melanoma cell proliferation and migration remains largely unknown. In the present study it was demonstrated that UCA1 expression was increased in melanoma tissues and cell lines. Inhibition of UCA1 expression markedly reduced melanoma cell migration and proliferation. Further investigation revealed that UCA1 functioned in melanoma cells through directly binding to miR-28-5p. The expression of miR-28-5p was significantly reduced in melanoma tissues and exhibited an inverse correlation with UCA1 expression.
Furthermore, HOXB3 was identified as a target gene of miR-28-5p in melanoma cells, and HOXB3 overexpression reversed the suppressive effects of UCA1 downregulation on melanoma cell proliferation and migration.

In recent years, an increasing number of lncRNAs have been demonstrated to be deregulated in human cancers, and some of these lncRNAs have tumor promoting or suppressive roles in melanoma $(10,23)$. For instance, the lncRNA H19 promotes glucose metabolism and cell growth in malignant melanoma via modulating the expression of miR-106a-5p and its target gene E2F3 (24). In contrast, the upregulation of lncRNA cancer susceptibility 2 has suppressive effects on the malignant progression of melanoma through regulating the miR-18a-5p/runt related transcription factor 1 axis (23). However, the molecular mechanism of lncRNAs in the regulation of melanoma cell proliferation and migration remains 
unknown. In the present study, it was demonstrated that the lncRNA UCA1 was significantly upregulated in a total of 21 melanoma tissues compared with adjacent non-tumor tissues. These findings were consistent with a previous report (14).

miRNAs, another type of non-coding small RNA that are 22-25 nucleotides in length, regulate the expression of their target genes through directly binding to the complementary sites in the 3'-UTR region of target mRNAs (25-27). Similar to $\operatorname{lncRNAs,~miRs~are~also~associated~with~various~}$ cancers (28-32). For instance, a previous study demonstrated that miR-33a-5p increased the radiosensitivity of melanoma cells by inhibition of glycolysis (33). In addition, it was also reported that miR-18b inhibited the growth of malignant melanoma via inhibition of hypoxia inducible factor- $1 \alpha$-mediated glycolysis (34). Through interacting with miRs, lncRNAs can regulate the expression of miRs and thus the downstream target genes $(35,36)$. For instance, the lncRNA X inactive specific transcript acts as a competing endogenous lncRNA to regulate transforming growth factor- $\beta 1$ by sponging miR-185 in gastric cancer (37). The lncRNA H19 promotes glucose metabolism and cell growth in malignant melanoma via inhibition of miR-106a-5p and thus upregulation of E2F3 (24). In the present study, it was demonstrated that UCA1 directly targets miR-28-5p, and inhibition of UCA1 enhanced the miR-28-5p expression. Furthermore, it was demonstrated that downregulation of miR-28-5p in melanoma tissues was inversely correlated with the upregulation of UCA 1 , which further suggests that increased UCA1 expression contributes to the decreased expression of miR-28-5p in melanoma. Further investigation results suggest that the oncogenic role of UCA1 in melanoma cells occurs through regulation of miR-28-5p.

Given that miRs function through regulating the expression of their target genes $(28,29)$, bioinformatics analysis was conducted to predict the potential target gene of miR-28-5p. We selected HOXB3 for the following studies. A previous study reported that miR-28-5p regulates HOXB3 expression in colorectal cancer cells (19), but the targeting relationship between miR-28-5p and HOXB3 has not been confirmed. In the present study, luciferase reporter gene assay results indicated that HOXB3 was a direct target gene of miR-28-5p in SK-MEL-28 cells. Furthermore, UCA1 downregulation reduced $\mathrm{HOXB} 3$ expression, which was rescued by knockdown of miR-28-5p in SK-MEL-28 cells. These findings suggest that UCA1 affects HOXB3 expression via miR-28-5p. Furthermore, it was demonstrated that HOXB3 overexpression significantly abolished the suppressive effects of UCA1 downregulation on SK-MEL-28 cell migration and proliferation, suggesting that UCA1 regulates SK-MEL-28 cell proliferation and migration via the miR-28-5p/HOXB3 axis.

In addition to the UCA1/miR-28-5p/HOXB3 axis, several other IncRNA/miRNA axes have been demonstrated to participate in the regulation of melanoma cell proliferation and migration. For instance, Chen et al (38) recently reported that IncRNA ILF3-AS1 promoted melanoma cell proliferation, migration, and invasion via negatively regulating miR-200b/a/429. Zhao et al (39) demonstrated that hepatocellular carcinoma upregulated EZH2-associated lncRNA enhanced the proliferation, migration and invasion of melanoma cells via inhibition of miR-200b/a/429. However, these studies did not investigate the downstream proteins of these axes. Therefore, the findings in the present study expand the understanding of the function of the $1 \mathrm{ncRNA} / \mathrm{miR} /$ protein pathways in melanoma cell proliferation and migration.

To the best of our knowledge, the present study is the first to report that the UCA1/miR-28-5p/HOXB3 axis has a key role in the regulation of melanoma cell proliferation and migration, which expands the understanding of the molecular mechanism underlying melanoma progression. The limitation of the present study is lack of animal experiments. Thus, future studies should further confirm the function of this signaling pathway in vivo.

\section{Acknowledgements}

Not applicable.

\section{Funding}

The present study was supported by New Xiangya Talent Project of the Third Xiangya Hospital of Central South University (grant no. JY201607).

\section{Availability of data and materials}

All data generated or analyzed during the present study are included in this published article.

\section{Authors' contributions}

$\mathrm{CH}$ wrote the manuscript. JZ designed the study and revised the manuscript. FT collected clinical tissues. CH, JC, DX, XL, YX and SW performed all experiments.

\section{Ethics approval and consent to participate}

The present study was approved by the Ethics Committee of Third Hospital of Central South University (Changsha, China). Written informed consent was obtained from all patients involved in the present study.

\section{Patient consent for publication}

Written informed consent was obtained from all patients involved in the present study.

\section{Competing interests}

The authors declare that they have no competing interests.

\section{References}

1. Trotter SC, Sroa N, Winkelmann RR, Olencki T and Bechtel M: A global review of melanoma follow-up guidelines. J Clin Aesthet Dermatol 6: 18-26, 2013.

2. Linos E, Swetter SM, Cockburn MG, Colditz GA and Clarke CA: Increasing burden of melanoma in the United States. J Invest Dermatol 129: 1666-1674, 2009.

3. Gao YL, Zhao ZS, Zhang MY, Han LJ, Dong YJ and Xu B: Long non-coding RNA PVT1 facilitates cervical cancer progression via negative regulating of miR-424. Oncol Res 25: 1391-1398, 2017.

4. Hua F, Li CH, Chen XG and Liu XP: Long Noncoding RNA CCAT2 knockdown suppresses tumorous progression by sponging mir-424 in epithelial ovarian vancer. Oncol Res 26: 241-247, 2018. 
5. Li J, Zi Y, Wang W and Li Y: LncRNA MEG3 inhibits cell proliferation and metastasis in chronic myeloid leukemia via targeting MiR-184. Oncol Res 26: 297-305, 2018.

6. Wang S, Hui Y, Li X and Jia Q: Silencing of lncRNA-CCDC26 restrains the growth and migration of glioma cells in vitro and in vivo via targeting miR-203. Oncol Res 26: 1143-1154, 2018.

7. Zhang JJ, Wang DD, Du CX and Wang Y: Long noncoding RNA ANRIL promotes cervical cancer development By acting as a sponge of miR-186. Oncol Res 26: 345-352, 2018.

8. Xiong W, Huang C, Deng H, Jian C, Zen C, Ye K, Zhong Z, Zhao X and Zhu L: Oncogenic non-coding RNA NEAT1 promotes the prostate cancer cell growth through the SRC3/IGF1R/AKT pathway. Int J Biochem Cell Biol 94: 125-132, 2018.

9. Cao L, Chen J, Ou B, Liu C, Zou Y and Chen Q: GAS5 knockdown reduces the chemo-sensitivity of non-small cell lung cancer (NSCLC) cell to cisplatin (DDP) through regulating miR-21/PTEN axis. Biomed Pharmacother 93: 570-579, 2017.

10. Chen L, Yang H, Xiao Y, Tang X, Li Y, Han Q, Fu J, Yang Y and Zhu Y: LncRNA GAS5 is a critical regulator of metastasis phenotype of melanoma cells and inhibits tumor growth in vivo. Onco Targets Ther 9: 4075-4087, 2016.

11. Li R, Zhang L, Jia L, Duan Y, Li Y, Bao L and Sha N: Long non-coding RNA BANCR promotes proliferation in malignant melanoma by regulating MAPK pathway activation. PLoS One 9: e100893, 2014

12. Luo J, Chen J, Li H, Yang Y, Yun H, Yang S and Mao X: LncRNA UCA1 promotes the invasion and EMT of bladder cancer cells by regulating the miR-143/HMGB1 pathway. Oncol Lett 14: $5556-5562,2017$

13. Bian Z, Jin L, Zhang J, Yin Y, Quan C, Hu Y, Feng Y, Liu H, Fei B, Mao Y, et al: LncRNA-UCA1 enhances cell proliferation and 5-fluorouracil resistance in colorectal cancer by inhibiting miR-204-5p. Sci Rep 6: 23892, 2016.

14. Tian Y, Zhang X, Hao Y, Fang Z and He Y: Potential roles of abnormally expressed long noncoding RNA UCA1 and malat-1 in metastasis of melanoma. Melanoma Res 24: 335-341, 2014.

15. Xue M, Pang H, Li X, Li H, Pan J and Chen W: Long noncoding RNA UCA1 promotes bladder cancer cell migration and invasion via hsa-miR-145/ ZEB1/2 /FSCN1 pathway. Cancer Sci 107: $18-27,2016$.

16. Fang Z, Zhao J, Xie W, Sun Q, Wang H and Qiao B: LncRNA UCA 1 promotes proliferation and cisplatin resistance of ora squamous cell carcinoma by sunppressing miR-184 expression. Cancer Med 6: 2897-2908, 2017.

17. Zhou Y, Chen Y, Ding W, Hua Z, Wang L, Zhu Y, Qian H and Dai T: LncRNA UCA1 impacts cell proliferation, invasion, and migration of pancreatic cancer through regulating miR-96/FOXO3. IUBMB Life 70: 276-290, 2018.

18. Wei Y, Sun Q, Zhao L, Wu J, Chen X, Wang Y, Zang W and Zhao G: LncRNA UCA1-miR-507-FOXM1 axis is involved in cell proliferation, invasion and G0/G1 cell cycle arrest in melanoma. Med Oncol 33: 88, 2016.

19. Almeida MI, Nicoloso MS, Zeng L, Ivan C, Spizzo R, Gafà R, Xiao L, Zhang X, Vannini I, Fanini F, et al: Strand-specific miR-28-5p and miR-28-3p have distinct effects in colorecta cancer cells. Gastroenterology 142: 886-896 e889, 2012

20. Xu J, Jiang N, Shi H, Zhao S, Yao S and Shen H: miR-28-5p promotes the development and progression of ovarian cancer through inhibition of N4BP1. Int J Oncol 50: 2236, 2017.

21. Livak KJ and Schmittgen TD: Analysis of relative gene expression data using real-time quantitative PCR and the 2(-Delta Delta C(T)) method. Methods 25: 402-408, 2001.

22. Li $\mathrm{F}$ and $\mathrm{Hu} \mathrm{CP}$ : Long non-coding RNA urothelial carcinoma associated 1 (UCA1): Insight into its role in human diseases. Crit Rev Eukaryot Gene Expr 25: 191-197, 2015.
23. Zhang Y, Qian W, Feng F, Cao Q, Li Y, Hou Y, Zhang L and Fan J: Upregulated lncRNA CASC2 may inhibit malignant melanoma development through regulating miR-18a-5p/RUNX1. Oncol Res: doi.org/10.3727/096504018X15178740729367.

24. Luan W, Zhou Z, Ni X, Xia Y, Wang J, Yan Y and Xu B: Long non-coding RNA H19 promotes glucose metabolism and cell growth in malignant melanoma via miR-106a-5p/E2F3 axis. J Cancer Res Clin Oncol 144: 531-542, 2018.

25. Li Z, Guo J, Ma Y, Lin Z and Zhang L: Oncogenic role of MicroRNA-30b-5p in glioblastoma through targeting proline-rich transmembrane protein 2. Oncol Res 26: 219-230, 2018.

26. Zhu H, Huang L, Zhu S, Li X, Li Z, Yu C and Yu X: Regulation of autophagy by systemic admission of microRNA-141 to target HMGB1 in 1-arginine-induced acute pancreatitis in vivo. Pancreatology 16: 337-346, 2016.

27. Zeng F, Xue M, Xiao T, Li Y, Xiao S, Jiang B and Ren C: MiR-200b promotes the cell proliferation and metastasis of cervical cancer by inhibiting FOXG1. Biomed Pharmacother 79: 294-301, 2016

28. Wang S, Cao KE, He Q, Yin Z and Zhou J: miR-199a-5p induces cell invasion by suppressing E-cadherin expression in cutaneous squamous cell carcinoma. Oncol Lett 12: 97-101, 2016.

29. Su Y,Xiong J,Hu J, Wei X,Zhang X and RaoL: MicroRNA-140-5p targets insulin like growth factor 2 mRNA binding protein 1 (IGF2BP1) to suppress cervical cancer growth and metastasis. Oncotarget 7: 68397-68411, 2016.

30. Zhou J, Xu D, Xie H, Tang J, Liu R, Li J, Wang S, Chen X, Su J, Zhou X, et al: miR-33a functions as a tumor suppressor in melanoma by targeting HIF-1 $\alpha$. Cancer Biol Ther 16: 846-855, 2015.

31. Zhou J, Liu R, Wang Y, Tang J, Tang S, Chen X, Xia K, Xiong W, $\mathrm{Xu} \mathrm{D}$, Wang S, et al: miR-199a-5p regulates the expression of metastasis-associated genes in B16F10 melanoma cells. Int J Clin Exp Pathol 7: 7182-7190, 2014

32. Zhou J, Liu R, Luo C, Zhou X, Xia K, Chen X, Zhou M, Zou Q, Cao P and Cao K: MiR-20a inhibits cutaneous squamous cell carcinoma metastasis and proliferation by directly targeting LIMK1. Cancer Biol Ther 15: 1340-1349, 2014.

33. Cao K, Li J, Chen J, Qian L, Wang A, Chen X, Xiong W, Tang J, Tang S, Chen Y, et al: microRNA-33a-5p increases radiosensitivity by inhibiting glycolysis in melanoma. Oncotarget 8 : 83660-83672, 2017.

34. Chen Y, Zhang Z, Luo C, Chen Z and Zhou J: MicroRNA-18b inhibits the growth of malignant melanoma via inhibition of HIF-1 $\alpha$-mediated glycolysis. Oncol Rep 36: 471-479, 2016.

35. Liu S, Song L, Zeng S and Zhang L: MALAT1-miR-124-RBG2 axis is involved in growth and invasion of HR-HPV-positive cervical cancer cells. Tumour Biol 37: 633-640, 2016.

36. Xie CH, Cao YM, Huang Y, Shi QW, Guo JH, Fan ZW, Li JG, Chen BW and Wu BY: Long non-coding RNA TUG1 contributes to tumorigenesis of human osteosarcoma by sponging miR-9-5p and regulating POU2F1 expression. Tumour Biol 37: 15031-15041, 2016.

37. Zhang Q, Chen B, Liu $\mathrm{P}$ and Yang J: XIST promotes gastric cancer (GC) progression through TGF-betal via targeting miR-185. J Cell Biochem 119: 2787-2796, 2018

38. Chen X, Liu S, Zhao X, Ma X, Gao G, Yu L, Yan D, Dong H and Sun W: Long noncoding RNA ILF3-AS1 promotes cell proliferation, migration, and invasion via negatively regulating miR-200b/a/429 in melanoma. Biosci Rep 37: BSR20171031, 2017.

39. Zhao H, Xing G, Wang Y, Luo Z, Liu G and Meng H: Long noncoding RNA HEIH promotes melanoma cell proliferation, migration and invasion via inhibition of miR-200b/a/429. Biosci Rep 37: BSR20170682, 2017. 